\title{
Biopsy site identified with FDG PET-CT for diagnosis of tuberculosis in a child
}

\author{
Pierre Goussard (10 , ${ }^{1}$ Celeste Burger, ${ }^{2}$ Helena Rabie, ${ }^{1}$ Savvas Andronikou ${ }^{3}$
}

${ }^{1}$ Paediatrics and Child Health, Stellenbosch University Faculty of Medicine and Health Sciences, Cape Town, Western Cape, South Africa

${ }^{2}$ Division of Nuclear Medicine, Stellenbosch University Faculty of Medicine and Health Sciences, Cape Town, Western Cape, South Africa

${ }^{3}$ Department of Pediatric Radiology, University of Pennsylvania, Philadelphia, Pennsylvania, USA

\section{Correspondence to} Professor Pierre Goussard; pgouss@sun.ac.za

Accepted 3 December 2021

\section{DESCRIPTION}

A 10-year-old, HIV-negative boy presented with significant weight loss, mild left hip arthralgia, a chronic nasal bridge ulcer and distal tracheal compression on chest X-ray. Biopsy of the nasal bridge ulcer was negative, and bronchoscopy was normal, although the Xpert MTB/RIF was trace positive. The child was started on anti-tuberculosis (TB) treatment based on constitutional symptoms, reactive Mantoux $(20 \mathrm{~mm})$ and the Xpert MTB/RIF result. Rheumatology and the immunology work-up was negative. Due to the inconclusive testing and ongoing symptoms which included fever, weight loss and persistent mild left hip arthralgia, F-18 fluorodeoxyglucose (FDG) Positron emission tomography (PET) and computed tomography (CT) (PET-CT) was performed to identify either intrathoracic or extrathoracic biopsy sites (preferably lymph nodes) to confirm a diagnosis of TB. The scan identified abscess formation in both psoas muscles with the activity extending from the left psoas muscle into the left gluteus maximus muscle. It also identified intense uptake in L1/L2 spondylodiscitis, and multilevel destructive changes in the vertebral bodies of L1, L2, L4, L5 and S1 with extension into the spinal canal at the lower two levels (figure 1). MRI of the lumbosacral spine confirmed the multifocal vertebral lesions (L1, L2, L5, S1) demonstrating signal alteration and height loss with relative preservation of the intervertebral discs, as well as predominantly prevertebral abscesses and subligamentous spread, typical of tuberculous spondylitis. There were also some posterior impressions on the thecal sac at S1 from the paraspinal abscess. Extensive soft tissue abscesses were present, involving the psoas muscle on the left and bilaterally the iliacus muscles, with deep extensions into the paravertebral muscles, extending subcutaneously on the left (figure 2).

Thick pus was aspirated from the psoas abscess under ultrasound guidance. This confirmed drugsensitive TB both on Xpert MTB/RIF and on culture.
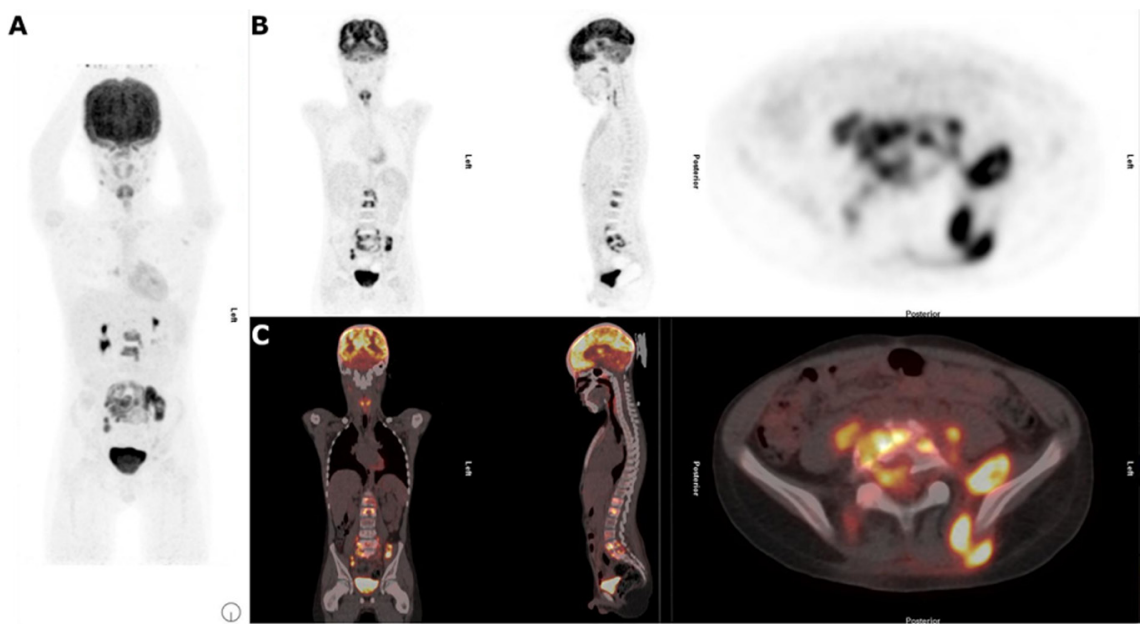

Figure 1 Positron emission tomography (PET) and computed tomography (CT) was performed 68 min after the administration of $97 \mathrm{MBq}$ of ${ }^{18} \mathrm{~F}$-fluorodeoxyglucose (FDG) on a Philips Gemini TF PET-CT scanner (Philips Medical Systems) and identified only limited pulmonary disease. In the chest, mild to moderate (maximum standardized uptake value (SUV max $_{3.32)}$ focal uptake in the upper and lower lobes of the right lung and in the lower lobe of the left lung, corresponded to low-density nodular opacities on CT (not shown), with several additional nodules being too small to characterise on PET. Extrapulmonary disease was much more extensive: intense uptake (SUV ${ }_{\max }$ 8.30) was observed in an L1/L2 spondylitis, with destructive changes in the L1 and L2 vertebral bodies. Similarly, intense uptake was seen in a destructive process involving the anterior aspects of L4, L5 and S1 (SUV ${ }_{\max }$ 10.27) —with abscess formation in bilateral psoas muscles and in the presacral region anteriorly, and extension into the spinal canal at the lower two levels posteriorly. Activity in the left psoas muscle was seen to track posteriorly into left quadratus lumborum, iliacus, longissimus thoracis, and finally into the medial aspect of the gluteus maximus muscle (SUV $\left.{ }_{\max } 14.14\right)$. On the right, there was posterior extension to involve the right longissimus thoracis muscle (SUV ${ }_{\max }$ 3.98). Intense focal uptake (SUV $_{\text {max }}$ 7.99) was also observed in right common and external iliac lymph node lesions. Moderate uptake $\left(S_{\text {SUV }}\right.$ 4.81) was also observed in the nasal region, corresponding to the known nasal bridge lesion with likely ethmoid sinus involvement. SUV max $_{\text {ax }}$ was measured on the standard $4 \mathrm{~mm}$ PET reconstruction. 


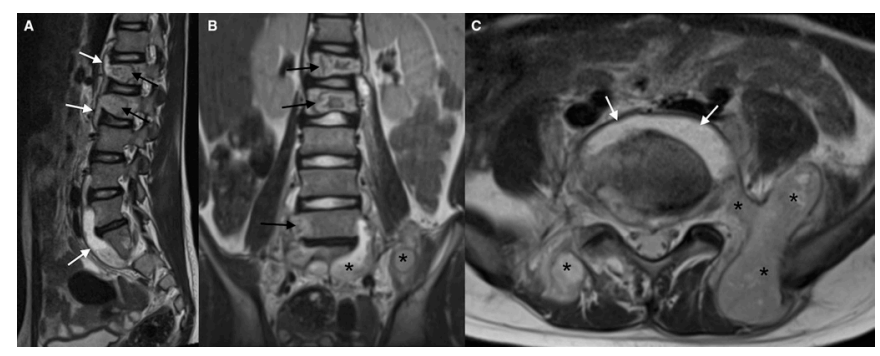

Figure 2 A-C) Sagittal (A), coronal (B) and axial (C) T2 weighted MRI of the lumbosacral spine demonstrating the multifocal vertebral lesions (L1, L2, L5, S1) with signal alteration and height loss (black arrows) but relative preservation of the intervertebral discs, as well as predominantly prevertebral abscesses (white arrows) and subligamentous spread, typical of tuberculous spondylitis. Extensive soft tissue abscesses are also demonstrated (asterisks), involving the psoas muscle on the left and bilaterally the iliacus muscles with deep extensions into the paravertebral muscles, extending subcutaneously on the left.

TB is the leading cause of death due to a single infectious agent globally with childhood TB accounting for $14 \%$ of the overall TB mortality worldwide. The difficulty in confirming TB in children microbiologically can result in delays in starting TB and also lead to more severe extensive disease.

F-18 FDG PET/CT is emerging as a valuable imaging tool in the management of complicated TB. ${ }^{1}$ While most of this benefit is supported by research in adults, there is growing recognition of PET/CTs utility in children. ${ }^{2}$ Advances in modern PET/CT scanner technology (higher sensitivity PET detectors, iterative CT reconstruction techniques) also continue to make such scanning safer in terms of administered radiation dose, to the advantage of paediatric patients. ${ }^{3}$

F-18 FDG PET/CT is highly sensitive for active pulmonary and extrapulmonary TB infection. ${ }^{14}$ The three-dimensional nature of PET/CT imaging (which can either be confined to a region of interest or include the whole body) enables the detection, accurate localisation and evaluation for extent of TB lesions.
In our patient, the advantage of using FDG PET/CT was the demonstration of disseminated TB and the identification of an abscess which could be aspirated to confirm the diagnosis. The findings of the FDG PET/CT correlated with the findings on MRI.

\section{Learning points}

- Challenges in diagnosing tuberculosis (TB) may result in delays in starting anti-TB treatment.

- F-18 fluorodeoxyglucose (FDG) PET/CT is highly sensitive for active pulmonary and extrapulmonary TB infection.

- FDG PET-CT can determine areas that can be either be biopsy or aspirated for diagnosis and microbiological conformation.

Acknowledgements Dr Alex Doruyter for his advice and help with manuscript.

Contributors $P G$ and $H R$ were involved with the clinical management of the patient. SA and CB were involved with the imaging of the patient. All were involved in creating the manuscript.

Funding The authors have not declared a specific grant for this research from any funding agency in the public, commercial or not-for-profit sectors.

Competing interests None declared.

Patient consent for publication Consent obtained from parent(s)/guardian(s).

Provenance and peer review Not commissioned; externally peer reviewed.

Case reports provide a valuable learning resource for the scientific community and can indicate areas of interest for future research. They should not be used in isolation to guide treatment choices or public health policy.

\section{ORCID iD}

Pierre Goussard http://orcid.org/0000-0003-1146-1307

\section{REFERENCES}

1 Jamar F, Buscombe J, Chiti A, et al. EANM/SNMMI Guideline for ${ }^{18} \mathrm{~F}$-FDG Use in Inflammation and Infection. J Nucl Med 2013;54:647-58.

2 Pelletier-Galarneau M, Martineau P, Zuckier LS, et al. 18 F-FDG-PET/CT imaging of thoracic and extrathoracic tuberculosis in children. Semin Nucl Med 2017;47:304-18.

3 Fahey FH, Goodkind A, MacDougall RD, et al. Operational and Dosimetric aspects of pediatric PET/CT. J Nucl Med 2017;58:1360-6.

4 Stelzmueller I, Huber $\mathrm{H}$, Wunn R, et al. 18F-Fdg PET/CT in the initial assessment and for follow-up in patients with tuberculosis. Clin Nucl Med 2016;41:e187-94.

Copyright 2021 BMJ Publishing Group. All rights reserved. For permission to reuse any of this content visit

https://www.bmj.com/company/products-services/rights-and-licensing/permissions/

BMJ Case Report Fellows may re-use this article for personal use and teaching without any further permission.

Become a Fellow of BMJ Case Reports today and you can:

- Submit as many cases as you like

- Enjoy fast sympathetic peer review and rapid publication of accepted articles

- Access all the published articles

- Re-use any of the published material for personal use and teaching without further permission

Customer Service

If you have any further queries about your subscription, please contact our customer services team on +44 (0) 2071111105 or via email at support@bmj.com.

Visit casereports.bmj.com for more articles like this and to become a Fellow 